\title{
Acute Onset Ocular Hypotony after Coronary Artery Bypass Surgery
}

\author{
Sarah Farukhi Ahmed Audrey Xi Tai Mason Schmutz John Combs \\ Sameh Mosaed \\ Gavin Herbert Eye Institute and University of California, Irvine School of Medicine, \\ Irvine, CA, USA
}

\section{Keywords}

Coronary artery bypass graft $\cdot$ Ischemic optic neuropathy $\cdot$ Ocular hypotony $\cdot$ Episcleral venous pressure

\begin{abstract}
Importance: The purpose of this case report is to evaluate risk factors associated with postcoronary artery bypass graft (CABG) ocular hypotony compared to post-CABG ischemic optic neuropathy. Observations: The patient described here is a single case at the University of California, Irvine Medical Center, from July 2016. This case demonstrates the rare incidence of acute post-CABG ocular hypotony and vision loss in a patient with prior history of optic atrophy. Both vision loss and hypotony resolved completely to baseline without intervention within 3 days postoperatively. Conclusions and Relevance: Severe anemia and large fluctuations in central venous pressure and blood pressure can occur in any patient undergoing CABG surgery. These hemodynamic shifts can cause transient ischemia to pressure controlling systems such as the ciliary body and reduce episcleral venous pressure. Other risk factors for acute hypotony in the setting of CABG surgery also include the use of hypertonic agents, cardiopulmonary bypass, and intravenous anesthesia.




\section{Introduction}

Ocular hypotony is defined as intraocular pressure (IOP) $<10 \mathrm{~mm} \mathrm{Hg}$ [1]. Clinically significant ocular hypotony, however, occurs when the low IOP results in an inability to maintain functionality of the eye [2]. This typically occurs at IOP $<5 \mathrm{~mm} \mathrm{Hg}$ [2]. Acute reduction of IOP in the absence of any intraocular pathology is rare.

In our case, we found clinically significant bilateral ocular hypotony within a few hours after the coronary artery bypass grafting (CABG) procedure. Drastic hemodynamic shifts during CABG can cause reduced blood flow to the ciliary body and decrease episcleral venous pressure [3]. In this setting of decreased aqueous production, the administration of intravenous anesthesia and hyperosmotic medications such as intravenous sodium bicarbonate can offset the balance of pressure homeostasis in the eye [4]. Interestingly for our patient, the hypotony resolved by postoperative day 1 with correction of the systemic blood pressure and arterial blood gas. No intervention was required to return to baseline IOP.

\section{Case Presentation}

Our patient was a 62-year-old male with a known history of hypertension and rightsided pituitary microadenoma status post-transsphenoidal resection 2 years prior to presentation. His pituitary adenoma had left him with bitemporal hemianopia and bilateral optic nerve atrophy (Fig. 1, Fig. 2). His baseline vision 1 month prior to surgery was 20/40 OD and count fingers OS. IOPs were documented within normal limits, $14 \mathrm{~mm} \mathrm{Hg} \mathrm{OU} \mathrm{[5].} \mathrm{The} \mathrm{optic}$ nerve cup-to-disc ratio was $0.5 \mathrm{OD}$ and $0.8 \mathrm{OS}$ with bilateral pallor.

After a positive exercise stress test and a subsequent cardiac angiogram demonstrating severe 3-vessel coronary artery disease, the patient underwent a coronary artery bypass surgery. Anesthesia was induced with fentanyl, midazolam, and propofol. The operative course was largely uneventful, and blood pressure fluctuations fell within normal range of CABG surgery [6]. Hypothermia was induced, and cardiopulmonary bypass (CPB) was used to maintain systemic oxygenation; body temperature was kept at average $35.5^{\circ} \mathrm{C}$. During this time, the patient's blood pressure fluctuated twice below the baseline mean arterial pressure (MAP) of 65. At these points, blood pressure dropped to 61/40 (MAP 47) for 10 min and 70/48 (MAP 55) for $5 \mathrm{~min}$. The patient's lowest central venous pressure was $5 \mathrm{~mm} \mathrm{Hg}$, which lasted $35 \mathrm{~min}$.

When resuscitating the patient out of $\mathrm{CPB}$, he had persistent metabolic acidosis. His $\mathrm{pH}$ dropped to a nadir of 7.22. A total of 3 amps or $75 \mathrm{~mL}$ of $8.4 \%$ sodium bicarbonate 2,000 $\mathrm{mOsm} / \mathrm{L}$ ) were used to allow for $\mathrm{pH}$ to return to physiologic levels, 7.36.

Postoperatively, the patient was still hypotensive; however, blood gases had normalized. He was placed on dobutamine, vasopressin, and Levophed to maintain MAP 65. Hemoglobin and hematocrit levels were 8.8 and $26.2 \mathrm{~g} / \mathrm{dL}$, respectively, both below normal limits. The partial pressure of oxygen $\left(\mathrm{paO}_{2}\right)$ dropped to a $68.2 \mathrm{~mm} \mathrm{Hg}$ (normal 80-104), which normalized after adjustment of patient's mechanical ventilation to $99.1 \mathrm{~mm} \mathrm{Hg}$.

An hour postoperatively, the patient's corneal surface appeared "wrinkled and deflated" to the monitoring nursing staff. Ophthalmic examination was performed at bedside. Visual acuity after extubation was 20/200 OD and count fingers OS. This was a notable decline in vision that the patient realized immediately after extubation anesthesia wore off. The IOP was immeasurable by Tono-Pen and soft to digital palpation. Through bedside portable slitlamp examination, the cornea appeared edematous with central Descemet's folds. The ante- 
rior chamber was diffusely flat with iridocorneal touch 360 degrees. Bedside dilated fundus examination was unremarkable by indirect ophthalmoscopy. There were no gross choroidal effusions. The retina was flat and attached 360 degrees with extramacular drusen OU (Fig. 3 ). A decision was made by the ophthalmology attending physician to re-examine the patient after normalization of systemic blood pressure and blood gases.

On postoperative day 1 , the patient noted a subjective improvement in his vision. His blood pressure normalized, and he was weaned off all vasopressive agents. Arterial blood gas monitoring demonstrated $\mathrm{paO}_{2} 118 \mathrm{~mm} \mathrm{Hg}$. Hemoglobin and hematocrit levels increased to 10.2 and $30.2 \mathrm{~g} / \mathrm{dL}$, respectively, after 1 unit of packed red blood cells. On ophthalmic examination, vision improved to 20/50 OD, count fingers OS. IOP was $11 \mathrm{~mm} \mathrm{Hg}$ OU. The corneal edema was still present with trace Descemet's folds. The anterior chamber was shallow with no iridocorneal touch. Dilated fundus examination was stable. Ophthalmic ultrasound confirmed the absence of any retinal detachments or choroidal effusions.

By postoperative day 2, the vision returned to full baseline 20/30 OD and count fingers OS. IOP was $13 \mathrm{~mm} \mathrm{Hg}$ OU. No corneal edema was appreciated, and the anterior chamber appeared well formed. Fundus examination was stable.

\section{Discussion}

IOP is a dynamic entity that fluctuates based on the homeostasis of aqueous humor inflow and outflow. In our case, the mechanism of acute IOP lowering is likely multifactorial resulting from intraoperative anesthesia, large fluctuations in systemic blood pressure and $\mathrm{paO}_{2}$, and administration of hyperosmotic sodium bicarbonate [4, 7].

In understanding the mechanism of this paradigm, a brief overview of the aqueous humor pathway is crucial. The aqueous humor production occurs at a rate of $2.0-2.5 \mathrm{uL} / \mathrm{min}$ [5]. It is achieved by 3 core pathways: (a) active secretion (against the pressure gradient), (b) ultrafiltration (along the pressure gradient), and (c) diffusion (along the concentration gradient). Typically, if IOP is below physiologic baseline, the ultrafiltration and diffusion gradients will increase aqueous production, while active secretion will continue at its basal rate. Thus, the system acts as a safety lock to prevent deviation from homeostasis [5].

On the other hand, ocular outflow occurs at a rate of $0.3 \mathrm{uL} / \mathrm{min} / \mathrm{mm} \mathrm{Hg}$ [5]. There are 2 pathways for outflow: uveoscleral and trabecular. Uveoscleral outflow occurs through a pressure-independent flow of fluid through the ciliary body and iris muscle root into the supraciliary and suprachoroidal space [8]. The fluid then exits through an intact sclera or along the vessels that penetrate it. Trabecular outflow is pressure dependent, meaning the aqueous flows down a pressure gradient. It involves flow of aqueous through the trabecular meshwork, through Schlemm's canal, into the episcleral veins, finally draining into the superior ophthalmic vein. Episcleral venous pressure can be affected by various internal and external factors. Both CVP and anesthesia medications have been shown to reduce episcleral venous pressure $[5,9]$.

A disruption in the mechanism of secretion or outflow will therefore result in a shift from ocular homeostasis [5]. In our scenario, the reduced $\mathrm{MAP}$ and $\mathrm{paO}_{2}$ likely resulted in global ischemia to the ciliary body causing reduced aqueous humor secretion. With diminished operative CVP and the administration of intraoperative anesthesia, episcleral venous pressure was also reduced [9]. Reduced episcleral venous pressure increases the pressure gradient down the trabecular outflow pathway, thereby increasing aqueous outflow. Thus, the overall effect is decreased aqueous humor production and increased aqueous outflow. 
CABG surgeries require a greater shift in hemodynamic flow as compared to most other vascular surgeries [7]. Extracorporeal circulation is required to maintain systemic perfusion intraoperatively. The aorta is cross-clamped, hypothermia is employed and systemic blood flow is redirected through a CPB pump [10]. This pump not only reduces systemic blood flow but also causes net hemodilution, resulting in reduced hemoglobin concentrations. Less oxygen is thus supplied to vascular structures such as the choroid, ciliary body, and retina [10].

Technically, these events occur during every CABG surgery; however, acute hypotony is a rare postsurgical complication [11]. Prior reports of post CABG ocular hypotony discuss its occurrence after administration of hypertonic medications such as mannitol [3]. This suggests that any medication with the potential to lower IOP can offset homeostasis in an already partially compromised setting. In our case, the patient was treated with 3 amps of $8.4 \%$ sodium bicarbonate. This agent has an osmolarity of 2,000 $\mathrm{mOsm} / \mathrm{L}$, which is seven times greater than serum osmolarity (285-295 mOsm/L) [4]. Physiologically, hyperosmolarity in the serum results in reduced aqueous secretion down the diffusion pathway [4]. The net effect is similar to a seesaw, in which each additional factor combined weighs against ocular homeostasis to finally offset the balance.

Of note, post-CABG permanent vision loss has also been reported in more common cases of perioperative ischemic optic neuropathy (ION) $[10,12]$. Perioperative ION can occur in the setting of any revised-cardiac-risk-index high-risk surgery, notably CABG procedures, spinal procedures, orthopedic surgeries, and radical neck dissections [11, 12]. Risk factors for development of perioperative ION all involve reduced blood flow to the optic nerve. These include hypotension, increased peripheral vascular resistance, anemia (reduced oxygen carrying capacity), and increased IOP [12].

Due to his unique history of prior optic nerve atrophy from pituitary adenoma, the patient's redundant nerve fibers had already been compromised. Any further damage to the optic nerve would have likely resulted in permanent irreversible vision loss. In fact, the absence of optic nerve damage and complete vision restoration in our patient after CABG is truly remarkable. Since elevated IOP is a known risk factor for perioperative ION, the patient's acute hypotony may have actually been protective against it, allowing for adequate perfusion to the optic nerve [12].

Based on these findings, vision assessment and ophthalmic examination after revisedcardiac-risk-index classified high-risk surgeries are important. Though these ocular sequelae are rare, increased vigilance can prevent serious ophthalmic complications. In cases of abnormal findings ocular pathology, an ophthalmology consultation should be considered.

\section{Acknowledgment}

This report was supported in part by the "Research to Blindness" grant.

\section{Statement of Ethics}

Members of the University of California, Irvine, are committed to the highest ethical standards. We recognize that we hold the University in trust for the people of the State of California. Our policies, procedures, and standards provide guidance for application of the ethical values stated below in our daily life and work as members of this community. 
Farukhi Ahmed et al.: Acute Onset Ocular Hypotony after Coronary Artery Bypass Surgery

\section{Disclosure Statement}

Authors have no proprietary interests and no financial disclosures.

\section{References}

1 American Academy of Ophthalmology: Uveoscleral outflow.

http://www.aao.org/bcscsnippetdetail.aspx?id=58b9c973-7a74-41ae-913f-0d8fc8d7dfb3.

2 Sanders, Sheila P, Evans J. Ocular hypotony. Updated May 6, 2016. http://emedicine.medscape.com.

-3 Kubo N, Sakuragi T, Takashi M, Tetsuya S, et al: Collapsed anterior chamber during hypothermic cardiopulmonary bypass. 1998. http://journals.lww.com/anesthesia-

analgesia/Fulltext/1998/04000/Collapsed_Anterior_Chamber_During_Hypothermic.59.asp.

-4 Bietti G, Virmo M, Pecori-Giraldi J: Acetozolamide, metabolic acidosis, and intraocular pressure. Am J Ophthalmol 1975;80:360-369.

5 Cantor, Loius B, Rapuano, CJ: Basic and clinical science course: Glaucoma, vol 10. New York, American Academy of Ophthalmology, 2015, Print. https://store.aao.org/clinical-education/product-line/basicand-clinical-science-course-

bcsc.html?product_line=Basic+and+Clinical+Science+Course+\%28BCSC\%29\&topic=Glaucoma.

6 International Anesthesia Research Society (IARS): "Variation in blood pressure during heart surgery linked to mortality risk." http://newswise.com/articles/variations-in-blood-pressure-during-heartsurgery-linked-to-mortality-risk. Article ID 5708082. Published June 23, 2011.

7 Aroesty JM, Aldea GS, Verrier E, Gersh BJ, Saperia GM: Patient education: coronary artery bypass graft surgery (beyond the basics). https://www.uptodate.com/contents/coronary-artery-bypass-graftsurgery-beyond-the-basics.

-8 Dorecka M, Miniewicz-Kurkowska J, Romaniuk D, et al: Anterior ischemic optic neuropathy after conventional coronary artery bypass graft surgery. Med Sci Monit 2011;17:CS70-CS74.

9 Cohn SL, Fleisher LA: Evaluation of cardiac risk prior to noncardiac surgery.

https://www.uptodate.com/contents/evaluation-of-cardiac-risk-prior-to-noncardiac-surgery.

10 Flanary WE, Phillips D, Kardon RH: Posterior ischemic optic neuropathy. April 3, 2015. http://EyeRounds.org/cases/212-PION.htm.

11 Lunn JK, Stanley TH, Eisele J, et al: High dose fentanyl anesthesia for coronary artery surgery: plasma fentanyl concentrations and influence of nitrous oxide on cardiovascular responses. Anesth Analg 1979;58:390-395.

12 Aminlari A, Callahan C: Medical, laser, and surgical management of inadvertent cyclodialysis cleft with hypotony. Arch Ophthalmol 2004;122:300-404. 


\section{Case Reports in Ophthalmology}

Case Rep Ophthalmol 2017;8:200-207

(c) 2017 The Author(s). Published by S. Karger AG, Basel www.karger.com/cop

OD

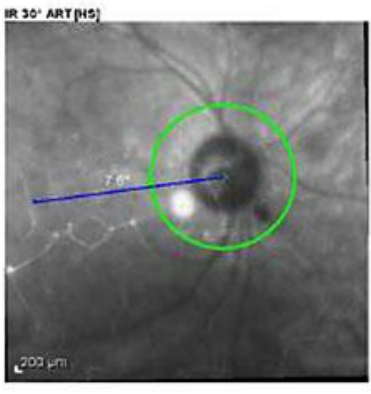
DOI: $10.1159 / 000468143$

Farukhi Ahmed et al.: Acute Onset Ocular Hypotony after Coronary Artery Bypass Surgery
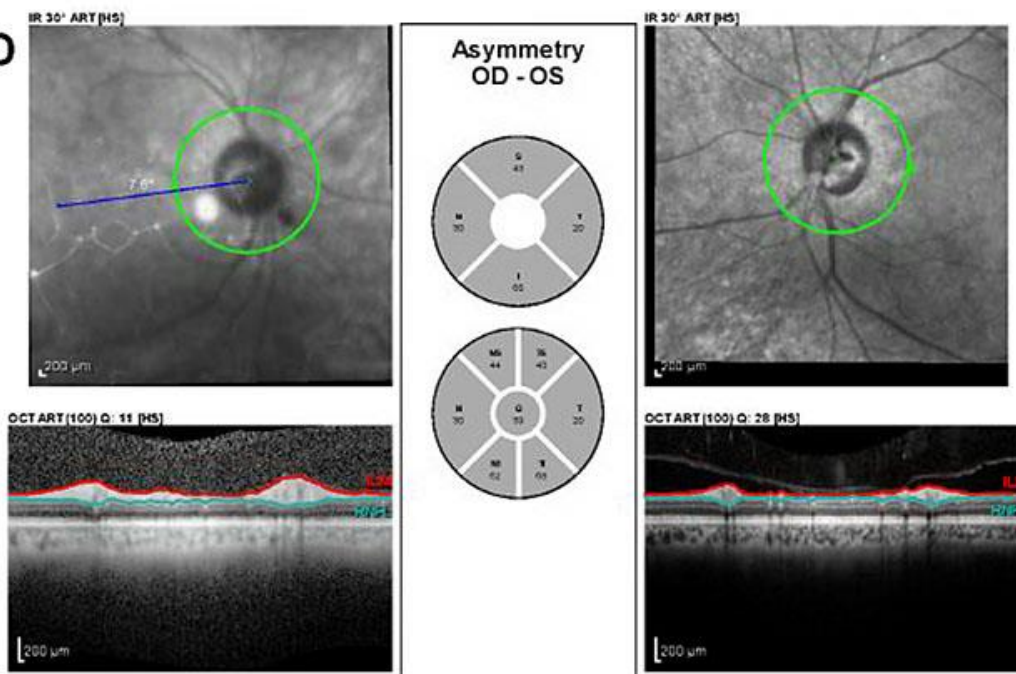

OS
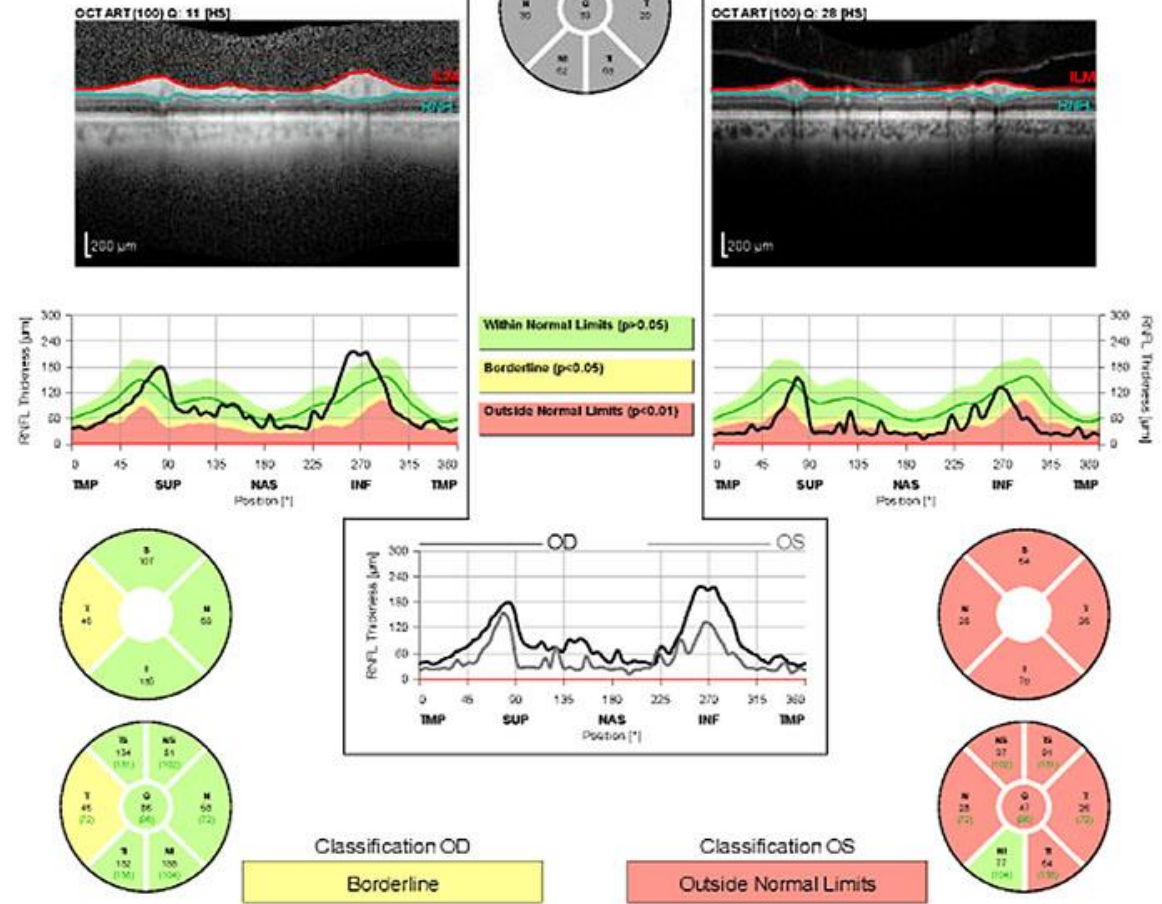

$-\infty$

os
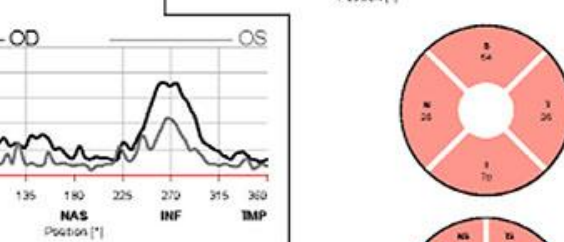

Classification OS

Outside Normal Limits



Fig. 1. Heidelberg optic coherence tomography showing optic atrophy in the left eye. 


\section{Case Reports in Ophthalmology}

Case Rep Ophthalmol 2017;8:200-207 www.karger.com/cop

Farukhi Ahmed et al.: Acute Onset Ocular Hypotony after Coronary Artery Bypass Surgery

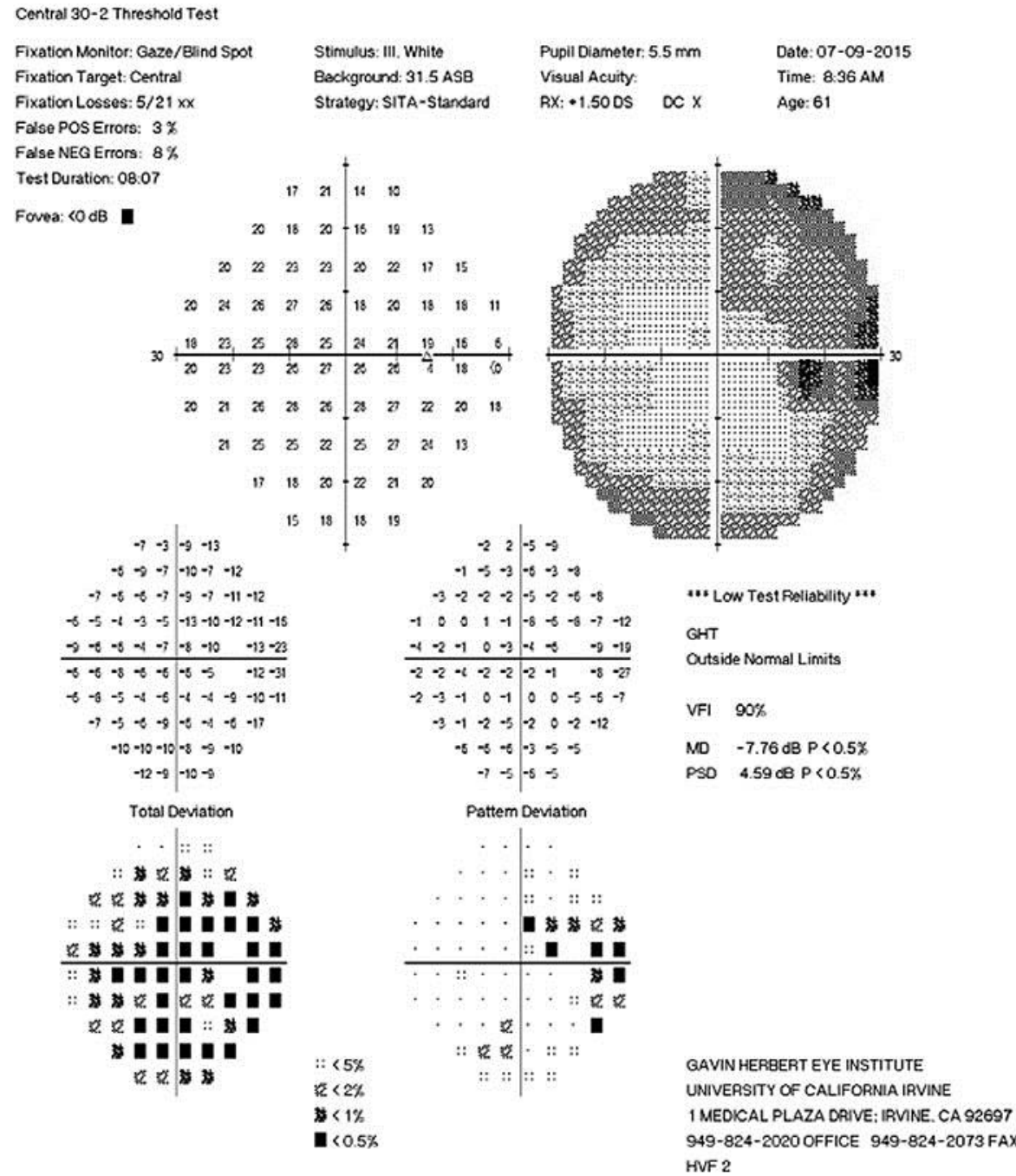

Fovea: $\angle O d B$

Stimulus: III. White

Beckground: 31.5 ASB

Strategy: SITA-Standard

Pupil Diameter: $5.5 \mathrm{~mm}$

Visual Acuity:

$R X: 1.500 S \propto C$

Date: 07-09-2015

Time: 8.36 AM

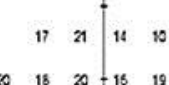

$\begin{array}{llllll}\infty & 18 & 20 & -15 & 19 & 13\end{array}$

$\begin{array}{llllllll}20 & 22 & 23 & 28 & 20 & 22 & 11 & 15\end{array}$

$\begin{array}{llllllllll}20 & 24 & 26 & 27 & 26 & 18 & 20 & 18 & 18 & 11\end{array}$



$\begin{array}{llllllll}a & z & z & 2 & z & z & a & 13\end{array}$



$15 \quad 18$ is 19

$\begin{array}{lll}-7 & -3 \mid-9 & -13\end{array}$

$-8 \rightarrow-\rightarrow-10-7-12$

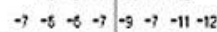

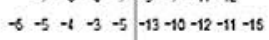

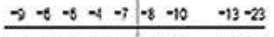

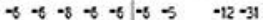

$-8-6-5-4-6-4<-4-9-10-11$

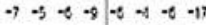

$-10-10-10-8 \Rightarrow-10$

$-12-9-10-8$

Total Deviation

. . |::: ::

::

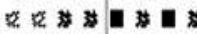

:: :

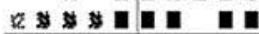

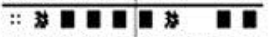

$\because:$ ฟ



औ 1010

हर 2

\begin{tabular}{ll|ll}
-2 & 2 & -5 & -9
\end{tabular}

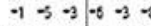

$-3-2-2-2-5-2-6-8$

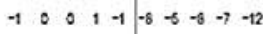





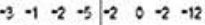

$-5=8-6-3=5-5$

$\rightarrow-5-5=5$

Pattem Deviation

:: :: ::: ::

GAVIN HERBERT EYE INSTITUTE

UNIVERSITY OF CALIFORNIA IRVINE

1 MEOICAL PLAZA DRIVE; IRVNE. CA 92697

949-824-2020 OFFICE 949-824-2073FAX

HVF 2

GrT

Outside Nomal Limits

VFI $90 \%$

MD $\quad-7.76 \mathrm{~dB} P<0.5 \%$

PSO $4.5908 P<0.5 \%$

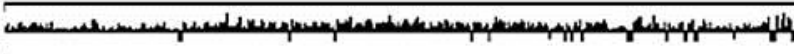

Q 2010 Car Zeiss Meditec

HFA II 750-40922-5.1.2/5.1.2

Fig. 2. Humphrey visual field 30-2 of the right eye showing baseline temporal visual field loss. The left eye could not be completed secondary to poor vision. 




Fig. 3. Dilated fundus examination showed diffuse extramacular drusen of the right eye. The macula was flat. There were no choroidal effusions. a Right eye optic nerve pallor with drusen inferior to the inferior arcade. $\mathbf{b}$ More right eye drusen.

Farukhi Ahmed et al.: Acute Onset Ocular Hypotony after Coronary Artery Bypass Surgery 